\title{
Dynamics and management of stage-structured fish stocks
}

Xinzhu Meng

Niklas L.P. Lundström

Mats Bodin

Åke Brännström (brnstrom@iiasa.ac.at)

\section{Approved by}

Ulf Dieckmann

Director, Evolution and Ecology Program

February 2015 Institute, its National Member Organizations, or other organizations supporting the work. 


\title{
Dynamics and management of stage-structured fish stocks
}

\author{
Xinzhu Meng \\ IceLab, Department of Mathematics and Mathematical Statistics, Umeå University, \\ SE-901 87 Umeå, Sweden \\ College of Information Science and Engineering, Shandong University of \\ Science and Technology, Qingdao 266510, P.R.China \\ Email address: xinzhu.meng@math.umu.se, mxz721106@sdust.edu.cn \\ Niklas L.P. Lundström \\ Department of Mathematics and Mathematical Statistics, Umeå University, \\ SE-901 87 Umeå, Sweden \\ Email address: niklas.lundstrom@math.umu.se \\ Mats Bodin \\ IceLab, Department of Ecology and Environmental Science, Umeå University, \\ SE-901 87 Umeå, Sweden \\ Email address: mats.bodin@emg.umu.se
}

Åke Brännström

IceLab, Department of Mathematics and Mathematical Statistics, Umeå University, SE-901 87 Umeå, Sweden

Evolution and Ecology Program, International Institute for Applied Systems Analysis, A-2361 Laxenburg, Austria

Email address: ake.brannstrom@math.umu.se

\begin{abstract}
With increasing fishing pressures having brought several stocks to the brink of collapse, there is a need for developing efficient harvesting methods that account for factors beyond merely yield or profit. We consider the dynamics and management of a stage-structured fish stock. Our work is based on a consumer-resource model which De Roos et al. (2008) have derived as an approximation of a physiologically-structured counterpart. First, we rigorously prove the existence of steady states in both models, that the models share the same steady states, and that there exists at most one positive steady state. Furthermore, we carry out numerical investigations which suggest that a steady state is globally stable if it is locally stable. Second, we consider multi-objective harvesting strategies which account for yield, profit, and
\end{abstract}


the recovery potential of the fish stock. The recovery potential is a measure of how quickly a fish stock can recover from a major disturbance and serves as an indication of the extinction risk associated with a harvesting strategy. Our analysis reveals that a small reduction in yield or profit allows for a disproportional increase in recovery potential. We also show that there exists a harvesting strategy with yield close to the maximum sustainable yield (MSY) and profit close to that associated with the maximum economic yield (MEY). In offering a good compromise between MSY and MEY, we believe that this harvesting strategy is preferable in most instances. Third, we consider the impact of harvesting on population size structure and analytically determine the most and least harmful harvesting strategies. We conclude that the most harmful harvesting strategy consists of harvesting both adults and juveniles, while harvesting only adults is the least harmful strategy. Finally, we find that a high percentage of juvenile biomass indicates elevated extinction risk and might therefore serve as an early-warning signal of impending stock collapse.

Keywords: Stage structure; Selective harvest; Pareto efficiency; MEY; MSY; Early-warning signal

\section{Introduction}

Mounting fishing pressure over the past decades has severely impacted aquatic ecosystems and marine resources (Garcia and Newton, 1995; Hutchings and Myers, 1994). Many fish stocks have been severely depleted and some have even collapsed. Few examples better highlight the dangers associated with intense exploitation than the Northern Cod Fishery at the eastern coast of Canada. In the early 1990s, the stock biomass suddenly collapsed to very low levels. Even though the Canadian government acted quickly to impose a moratorium on further exploitation, the stock remains commercially extinct to this date. The failure of the Northern Cod Fishery underlines the need for developing sustainable fisheries-management practices (Olsen et al., 2004). Theoretical models that account for both economic and ecological aspects of fisheries are likely to play a key role in this important endeavor.

Much of fisheries-management theory is based on simple unstructured models, often formulated with ordinary differential equations. Over the last three decades or so, there has been a trend towards increased realism in ecological theory. This trend has been supported by the introduction of physiologically-structured population models (e.g., Metz and Diekmann, 1986; Cushing, 1989; De Roos, 1997; Persson et al., 1998; Claessen et al., 2003; Farkas and Hagen, 2007). Whereas unstructured population models treat all individuals as equals, physiologically-structured population models distinguish between individuals based on one or more physiological characteristics. The size of an individual is a common choice in the context of fisheries, as individual size is often a good predictor of life-history state and associated vital rates such as mortality, fecundity, and growth.

Size-structured models are more difficult to work with than their unstructured counterparts, 
both analytically and computationally. In addition, they do not always connect with the existing fisheries-management theory in a straightforward way. While some physiologically-structured populations models can be reduced to finite-dimensional ODE models (e.g., Diekmann and Metz, 1989), this depends on the vital rates having a precise mathematical form. Recently, De Roos et al. (2008) formulated a stage-structured population model which incorporates key individual life-history processes. As this model was derived as an approximation of an ecologically motivated physiologically-structured population model, it is a promising candidate for a standard model of ecological dynamics in fisheries management. In Sect. 2, we present this physiologically-structured population model and show how the approximating stage-structured counterpart is derived. We then consider and, when possible, rigorously analyze the existence and stability of steady states in Sect. 3. In this section, we also introduce the recovery potential as a promising measure of a fish stocks ability to resist collapse. A reader who is not interested in the mathematical details could leave Sect. 3 for a later reading.

An important reason why physiologically-structured population models are preferable in fisheries management is that they allow more realistic description of harvesting regimes. Excess mortality induced by fisheries is often size- or stage-dependent. This dependence can arise from the choice of gear or fishing ground, from regulations stipulating size limits, or as an adaptation of the fisheries to size-dependent market prices. Although it has been known for some time that fishing induces changes in both abundance and size structure (see, e.g., Rijnsdorp et al.,1996; Olsen et al., 2004; Walsh et al., 2006), much remains to be understood about the consequences of size-dependent fishing mortality.

In Sect. 4, we consider the impact on fisheries yield, fisheries profit, and the recovery potential of the targeted fish stock. We further explore demographic effects of stage-selective harvesting. In particular, we study how different harvesting rates of juveniles and adults affect the size-composition and recovery potential of the fish stock. Finally, in the concluding Sect. 5, we discuss the results obtained and identify key challenges for future research.

\section{$2 \quad$ Model and parameters}

In this section, we recapitulate selected achievements by De Roos et al. (2008). We introduce their physiologically-structured population model and show how the approximative stage-structured counterpart is derived when harvesting is stage-selective. We also state the parameter values that we use in numerical investigations.

\section{$2.1 \quad$ Model}

We adopt a physiologically-structured population model formulated by De Roos et al. (2008), which accounts for key aspects of individual life histories. Below, we give a complete but brief 
description of the model elements and refer the reader to De Roos et al. (2008) for further details and motivations.

The population is composed of juveniles and adults which forage on a shared resource $R=R(t)$. Juveniles use all available energy for growth and maturation, while adults do not grow and instead invest all their energy in reproduction. Individuals are completely characterized by their size (weight) $s$. Juveniles are born with size $s_{0}$ and grow until they reach the size $s_{\mathrm{m}}$ at which they cease to grow, mature, and become adults. The juveniles' growth rates depend on resource abundance. Both foraging ability and metabolic requirements are assumed to increase with individual body size.

Let $n(s, t)$ be the size distribution of juvenile consumers with $s_{0} \leq s<s_{\mathrm{m}}$ and $N=N(t)$ the total number of adult consumers, all of size $s=s_{\mathrm{m}}$. The consumer-resource system with stage-selective harvesting is described by the following differential equations:

$$
\begin{aligned}
\frac{\partial}{\partial t} n(s, t)+\frac{\partial}{\partial s}(g(s, R) n(s, t)) & =-d_{\mathrm{j}}(R) n(s, t)-H_{\mathrm{j}}(s, n(s, t)), \quad \text { for } s_{0} \leq s<s_{\mathrm{m}}, \\
g\left(s_{0}, R\right) n\left(s_{0}, t\right) & =b\left(s_{\mathrm{m}}, R\right) N(t), \\
\frac{d}{d t} N(t) & =g\left(s_{\mathrm{m}}, R\right) n\left(s_{\mathrm{m}}, t\right)-d_{\mathrm{a}}(R) N(t)-H_{\mathrm{a}}(N), \\
\frac{d}{d t} R(t) & =G(R)-\left(\int_{s_{0}}^{s_{\mathrm{m}}} f_{\mathrm{j}}(s, R) n(s, t) d s+f_{\mathrm{a}}\left(s_{\mathrm{m}}, R\right) N(t)\right) .
\end{aligned}
$$

Here, $n\left(s_{\mathrm{m}}, t\right)=\lim _{s \rightarrow s_{\mathrm{m}}^{-}} n(s, t), g(s, R)$ represents the juvenile growth rate in body mass, $b\left(R, s_{\mathrm{m}}\right)$ is the fecundity rate of adults with $s_{\mathrm{m}}$, while $d_{\mathrm{j}}(R)>0$ and $d_{\mathrm{a}}(R)>0$ are the respective juvenile and adult background mortality rates. $H_{\mathrm{j}}(s, n(s, t))$ and $H_{\mathrm{a}}(N)$ represent the size-dependent respective harvesting rates of juveniles and adults, $f_{\mathrm{j}}(s, R)$ and $f_{\mathrm{a}}\left(s_{\mathrm{m}}, R\right)$ are the respective feeding rates of juveniles and adults, and $G(R)$ is the rate at which the resource grows in the absence of consumers.

The system above can be simplified by assuming that the juvenile and adult background mortality rates are constant and given by

$$
d_{\mathrm{j}}(R)=d_{\mathrm{j}}>0 \quad \text { and } \quad d_{\mathrm{a}}(R)=d_{\mathrm{a}}>0 .
$$

Additionally, it is assumed that juveniles and adults do not produce biomass when the energy intake is insufficient to cover maintenance requirements. The net biomass production per unit biomass of juveniles and adults, respectively, denoted by $w_{\mathrm{j}}(R)$ and $w_{\mathrm{a}}(R)$, are given by

$$
\begin{aligned}
& w_{\mathrm{j}}(R)= \begin{cases}\sigma I_{\max } \frac{R}{H+R}-T & \text { if } R>\frac{H}{\sigma I_{\max } / T-1}=R_{\mathrm{jT}}, \\
0 & \text { otherwise }\end{cases} \\
& w_{\mathrm{a}}(R)= \begin{cases}\sigma q I_{\max } \frac{R}{H+R}-T & \text { if } R>\frac{H}{\sigma q I_{\max } / T-1}=R_{\mathrm{aT}} . \\
0 & \text { otherwise }\end{cases}
\end{aligned}
$$


Here, $\sigma$ represents the efficiency of resource ingestion. The maximum ingestion rates per unit biomass of juveniles and adults are $I_{\max }$ and $q I_{\max }$, respectively. The factor $q$ represents the difference in ingestion rates between the juvenile stage and the adult stage. A value of $q<1$ implies that juveniles have a higher resource intake rate per unit body mass than the adults, whereas $q>1$ describe the opposite situation of adults having the higher ingestion rate. When $q=1$, juveniles and adults are equally able to ingest the resource. The juvenile growth rate, $g(s, R)$, and the adult birth rate, $b\left(R, s_{\mathrm{m}}\right)$, are defined as follows:

$$
g(s, R)=s w_{\mathrm{j}}(R) \quad \text { and } \quad b\left(s_{\mathrm{m}}, R\right)=\frac{s_{\mathrm{m}}}{s_{0}} w_{\mathrm{a}}(R) .
$$

The feeding rates of juveniles and adults are respectively given by

$$
f_{\mathrm{j}}(s, R)=s I_{\max } \frac{R}{H+R} \quad \text { and } \quad f_{\mathrm{a}}\left(s_{\mathrm{m}}, R\right)=s_{\mathrm{m}} q I_{\max } \frac{R}{H+R} .
$$

The resource follows semi-chemostat dynamics,

$$
G(R)=r\left(R_{\max }-R\right),
$$

in which $r$ denotes the intrinsic turnover rate and $R_{\max }$ denotes the maximum resource density in the absence of consumers.

Finally, the harvesting rates of juveniles and adults are assumed to be constant per capita:

$$
H_{\mathrm{j}}(s, n(s, t))=h_{\mathrm{j}} n(s, t) \quad \text { and } \quad H_{\mathrm{a}}(N)=h_{\mathrm{a}} N(t) .
$$

As first demonstrated by De Roos et al. (2008), the size-stage-structured population model described above can be simplified to an approximative stage-structured counterpart. This stagestructured model is obtained from System (1) by assuming that the distribution of the juveniles is fixed to its equilibrium distribution. Specifically, we assume that

$$
\frac{\partial}{\partial t} n(s, t)=0
$$

for all $s$ and $t$. Solving the PDE in System (1) for its equilibrium distribution yields

$$
n(s)=n\left(s_{0}\right)\left(\frac{s}{s_{0}}\right)^{-\left(1+\frac{d_{\mathrm{j}}+h_{\mathrm{j}}}{w_{\mathrm{j}}(R)}\right)} .
$$

To arrive at the stage-structured system, we introduce the variables

$$
J(t)=\int_{s_{0}}^{s_{\mathrm{m}}} s n(s, t) d s, \quad \text { and } \quad A(t)=s_{\mathrm{m}} N(t),
$$

and proceed as in De Roos et al. (2008; Eqs. 12-20) to obtain the simplified system:

$$
\begin{aligned}
\frac{d J}{d t} & =w_{\mathrm{a}}(R) A+\left(w_{\mathrm{j}}(R)-v\left(w_{\mathrm{j}}(R)\right)-d_{\mathrm{j}}-h_{\mathrm{j}}\right) J, \\
\frac{d A}{d t} & =v\left(w_{\mathrm{j}}(R)\right) J-\left(d_{\mathrm{a}}+h_{\mathrm{a}}\right) A, \\
\frac{d R}{d t} & =r\left(R_{\max }-R\right)-I_{\max } \frac{R}{H+R}(J+q A),
\end{aligned}
$$


in which

$$
v(x)=\frac{x-d_{\mathrm{j}}-h_{\mathrm{j}}}{1-\left(s_{0} / s_{\mathrm{m}}\right)^{1-\left(d_{\mathrm{j}}+h_{\mathrm{j}}\right) / x}} .
$$

The function $v(x)$ has the same sign as $x$ and a removable discontinuity at $x=d_{\mathrm{j}}+h_{\mathrm{j}}$. To make $v(x)$ continuous, we define $v\left(d_{\mathrm{j}}+h_{\mathrm{j}}\right)=\lim _{x \rightarrow d_{\mathrm{j}}+h_{\mathrm{j}}} v(x)=-\left(d_{\mathrm{j}}+h_{\mathrm{j}}\right) / \ln \left(s_{0} / s_{\mathrm{m}}\right)$.

Two major advantages of the stage-structured System (9) over the physiologically-structured System (1) are enhanced analytical tractability and reduced computational demands. In Sect. 3, we give rigorous proofs showing that both systems are uniformly bounded and that they have the same equilibria.

\subsection{Scaling and parametrization}

Table 1: Ecological and economic parameters

\begin{tabular}{|c|c|c|c|}
\hline Symbol & Value & Unit & Description \\
\hline$H$ & 1 & $\mathrm{~kg} / \mathrm{a} . \mathrm{u}$. & Half-saturation constant of the consumers \\
\hline$T$ & 1 & day $^{-1}$ & Maintenance rate \\
\hline$r$ & 1 & day $^{-1}$ & Resource turn-over and per capita growth rate \\
\hline$R_{\max }$ & 2 & $\mathrm{~kg} / \mathrm{a} . \mathrm{u}$ & The maximum resource density \\
\hline$\sigma$ & 0.5 & - & Efficiency of ingested biomass \\
\hline$s_{0}$ & 0.1 & $\mathrm{~cm}$ & Size at birth \\
\hline$s_{\mathrm{m}}$ & 10 & $\mathrm{~cm}$ & Size at maturation \\
\hline$I_{\max }$ & 10 & day $^{-1}$ & Maximum ingestion rates per unit biomass \\
\hline$q$ & 0.8 & - & Differences in ingestion ability between juveniles and adults \\
\hline$d_{\mathrm{j}}$ & 0.1 & day $^{-1}$ & Juvenile mortality \\
\hline$d_{\mathrm{a}}$ & 0.1 & day $^{-1}$ & Adult mortality \\
\hline$h_{\mathrm{j}}$ & - & day $^{-1}$ & Harvest rate of juveniles \\
\hline$h_{\mathrm{a}}$ & - & day $^{-1}$ & Harvest rate of adults \\
\hline$p_{\mathrm{j}}$ & 1.2 & $€ / \mathrm{kg}$ & Price of juvenile fish \\
\hline$p_{\mathrm{a}}$ & 6 & $€ / \mathrm{kg}$ & Price of adult fish \\
\hline$c_{\mathrm{j}}$ & 0.31 & $€ /$ a.u. & Costs of juvenile fish per one unit of effort \\
\hline$c_{\mathrm{a}}$ & 0.54 & $€ /$ a.u. & Costs of adult fish per one unit of effort \\
\hline
\end{tabular}

Note: All ecological parameters except $h_{\mathrm{j}}$ and $h_{\mathrm{a}}$ concern individual-level processes.

In Sects. 3-4 we carry out numerically investigations for varying stage-dependent harvesting rates $h_{\mathrm{j}}$ and $h_{\mathrm{a}}$. Table 1 gives the parameter values that are used. All ecological parameters are taken from De Roos et al. (2008) and are based on empirical data, with the exception of $R_{\max }=2$. For the body-mass-specific factor $q$ which phenomenologically describe the difference in resource exploitation between juveniles and adults, we adopt the value $q=0.8$. The half-saturation constant $H$ in the feeding response (5) express a resource density in kilogram biomass per unit area. Changing the value of $H$ can be interpreted as a change of the area in which the resource 
density and population density are expressed. By adopting the value $H=1$ from De Roos (2008) we thus effectively fix this area, which we refer to as an area unit (a.u.).

That the densities are expressed in an unknown area unit complicates the choice of economical parameters. However, in general, small juveniles fish has a lower economic value per kilogram than large adult fish, so we assume the value $p_{j}=1.2 € /$ kilogram and $p_{a}=6 € /$ kilogram as the price of juvenile and adult fish, respectively. To motivate our choice of cost parameters $c_{j}$ and $c_{a}$, we believe that the cost of harvesting relative the income should be fairly low in a pristine environment, where $15 \%$ might be a reasonable choice. Assuming a juveniles versus adult harvest ratio of $1: 4$ and adopting $c_{j}=0.31 € /$ a.u. and $c_{a}=0.54 € /$ a.u. as the cost per one unit of effort; the cost versus income ratio in a pristine environment will be approximately $15 \%$.

\section{Dynamics of stage-structured fish stocks}

In this section, we present results about the dynamics of the physiologically-structured population model, System (1), and its approximative stage-structured counterpart, (9). We first give rigorous proofs showing that both systems are uniformly bounded and that the two systems have the same equilibria under assumptions (2)-(7). We then introduce the measure of stock recovery potential, which is used in Sect. 4. Finally, a numerical investigation is carried out which indicates that System (9) always has exactly one global attractor. The global attractor is either an extinction equilibrium or a positive (interior) equilibrium.

\subsection{Existence of a global attractor}

The following theorem states that both System (1) and System (9) are uniformly bounded, i.e., for each system there exists a global attractor. For System (1), we do not need assumptions (2)-(7). The only assumptions needed to prove the existence of a global attractor for System (1) are the following: The resource and its growth rate are bounded, i.e., there exist constants $G_{\mathrm{m}}$ and $R_{\mathrm{m}}$ such that

$$
R(t) \leq R_{\mathrm{m}} \quad \text { and } \quad G(R) \leq G_{\mathrm{m}} .
$$

The harvest is non-negative and foraging rates for juveniles and adults are larger than the growth and fecundity rates, that is,

$$
0 \leq H_{\mathrm{j}}(s, n(s, t)), \quad 0 \leq H_{\mathrm{a}}(N), \quad g(s, R) \leq f_{\mathrm{j}}(s, R) \quad \text { and } \quad b(s, R) \leq f_{\mathrm{a}}(s, R) .
$$

Before stating the theorem, we point out that these assumptions are clearly weaker than (2)-(7). Note that if (12) fails then $J(t)+A(t)+R(t)$ may diverge to infinity, since the population biomass would possibly increase more than the decrease in resource biomass.

Theorem 3.1. If (11) and (12) hold, then System (1) is uniformly bounded. Moreover, System (9) is uniformly bounded. 
Proof. For System (1), define

$$
V(t)=\int_{s_{0}}^{s_{\mathrm{m}}} s n(s, t) d s+s_{\mathrm{m}} N(t)+R(t) .
$$

We prove the conclusion for System (1) by showing that $V(t)$ is bounded. From System (1) and by (12), we obtain

$$
\begin{aligned}
\frac{d V(t)}{d t}= & \int_{s_{0}}^{s_{\mathrm{m}}} s \frac{\partial}{\partial t} n(s, t) d s+s_{\mathrm{m}} \frac{\partial}{\partial t} N(t)+\frac{\partial}{\partial t} R(t) \\
\leq & -\int_{s_{0}}^{s_{\mathrm{m}}} s \frac{\partial}{\partial s}(g(s, R) n(s, t)) d s-d_{\mathrm{j}}(R) \int_{s_{0}}^{s_{\mathrm{m}}} s n(s, t) d s \\
& +s_{\mathrm{m}} g\left(s_{\mathrm{m}}, R\right) n\left(s_{\mathrm{m}}, t\right)-s_{\mathrm{m}} d_{\mathrm{a}}(R) N(t) \\
& +G(R)-\left(\int_{s_{0}}^{s_{\mathrm{m}}} f_{\mathrm{j}}(s, R) n(s, t) d s+f_{\mathrm{a}}\left(s_{\mathrm{m}}, R\right) N(t)\right) \\
= & s_{0} b\left(s_{\mathrm{m}}, R\right) N(t)+\int_{s_{0}}^{s_{\mathrm{m}}} g(s, R) n(s, t) d s-d_{\mathrm{j}}(R) \int_{s_{0}}^{s_{\mathrm{m}}} s n(s, t) d s-s_{\mathrm{m}} d_{\mathrm{a}}(R) N(t) . \\
& +G(R)-\left(\int_{s_{0}}^{s_{\mathrm{m}}} f_{\mathrm{j}}(s, R) n(s, t) d s+f_{\mathrm{a}}\left(s_{\mathrm{m}}, R\right) N(t)\right) .
\end{aligned}
$$

Now by (11) and (12), we have

$$
\begin{aligned}
\frac{d V(t)}{d t} & \leq G_{\mathrm{m}}-d_{\mathrm{j}}(R) \int_{s_{0}}^{s_{\mathrm{m}}} s n(s, t) d s-s_{\mathrm{m}} d_{\mathrm{a}}(R) N(t) \\
& \leq G_{\mathrm{m}}+R_{\mathrm{m}}-d_{\mathrm{j}}(R) \int_{s_{0}}^{s_{\mathrm{m}}} s n(s, t) d s-s_{\mathrm{m}} d_{\mathrm{a}}(R) N(t)-R(t) \\
& \leq G_{\mathrm{m}}+R_{\mathrm{m}}-\min _{R}\left\{d_{\mathrm{j}}(R), d_{\mathrm{a}}(R), 1\right\} V(t) .
\end{aligned}
$$

Thus, $\operatorname{since} \min _{R} d_{\mathrm{j}}(R)>0$ and $\min _{R} d_{\mathrm{a}}(R)>0$,

$$
V(t)<\frac{G_{\mathrm{m}}+R_{\mathrm{m}}}{\min _{R}\left\{d_{\mathrm{j}}(R), d_{\mathrm{a}}(R), 1\right\}}+\varepsilon
$$

for large enough $t$ and any $\varepsilon>0$. Hence, System (1) is uniformly bounded.

For System (9), we define

$$
V(t)=R(t)+J(t)+A(t),
$$


and hence

$$
\begin{aligned}
\frac{d V(t)}{d t} & =r\left(R_{\max }-R\right)-I_{\max } \frac{R}{R+H}(J+q A) \\
& +\left(w_{a}(R)-d_{\mathrm{a}}-h_{\mathrm{a}}\right) A+\left(w_{\mathrm{j}}(R)-d_{\mathrm{j}}-h_{\mathrm{j}}\right) J \\
& =r\left(R_{\max }-R\right) \\
& -\left((1-\sigma) I_{\max } \frac{R}{R+H}+T+d_{\mathrm{j}}+h_{\mathrm{j}}\right) J-\left((1-\sigma) q I_{\max } \frac{R}{R+H}+T+d_{\mathrm{a}}+h_{\mathrm{a}}\right) A \\
& \leq r R_{\max } \\
& -\min _{R}\left\{(1-\sigma) I_{\max } \frac{R}{R+H}+T+d_{\mathrm{j}}+h_{\mathrm{j}},(1-\sigma) q I_{\max } \frac{R}{R+H}+T+d_{\mathrm{a}}+h_{\mathrm{j}}, r\right\} V(t) .
\end{aligned}
$$

Since $\sigma<1$ and since $d_{\mathrm{j}}, d_{\mathrm{a}}, h_{\mathrm{j}}, h_{\mathrm{a}}, r$, and $T$ are positive, the theorem now follows as in the former case.

\subsection{Existence of equilibria}

We now explore the existence of equilibria to System (1) and (9). First note that if $w_{\mathrm{j}}(R)=0$, then $n(s, t)=0, N(t)=0$ and $R(t)=R_{\max }$, which implies that System (1) has an extinction equilibrium at $(J, A, R)=\left(0,0, R_{\max }\right)$. Therefore, in the rest of the paper we assume, in addition to $(2)-(7)$, that $w_{\mathrm{j}}(R)>0$, i.e., $R_{\mathrm{jT}}<R<R_{\max }$.

Theorem 3.2. System (1) has an extinction equilibrium $\left(0,0, R_{\max }\right)$ and at most one positive equilibrium $(\mathcal{J}, \mathcal{A}, \mathcal{R})$ where

$$
\mathcal{J}=\frac{n\left(s_{0}\right) s_{0}^{2}}{1-\frac{d_{j}+h_{j}}{w_{j}(R)}}\left(\left(\frac{s_{m}}{s_{0}}\right)^{1-\frac{d_{j}+h_{j}}{w_{j}(R)}}-1\right), \quad \mathcal{A}=\frac{n\left(s_{0}\right) s_{0}^{2} w_{j}(\mathcal{R})}{d_{a}+h_{a}}\left(\frac{s_{m}}{s_{0}}\right)^{1-\frac{d_{j}+h_{j}}{w_{j}(R)}}
$$

and $\mathcal{R}$ and $n\left(s_{0}\right)$ are obtained sequentially as the roots of the Eqs. (16) and (19).

Proof. It is clear that System (1) always has the extinction equilibrium $\left(0,0, R_{\max }\right)$. For a positive equilibrium, we solve the equations $\partial n / \partial t=0, d N / d t=0$ and $d R / d t=0$. We obtain, with the notation $n(s, t)=n(s)$,

$$
\begin{aligned}
w_{\mathrm{j}}(R) \frac{d(s n(s))}{d s} & =-d_{\mathrm{j}} n(s)-h_{\mathrm{j}} n(s), \\
s_{0} w_{\mathrm{j}}(R) n\left(s_{0}\right) & =\frac{s_{\mathrm{m}}}{s_{0}} w_{\mathrm{a}}(R) N, \\
0 & =s_{\mathrm{m}} w_{\mathrm{j}}(R) n\left(s_{\mathrm{m}}\right)-\left(d_{\mathrm{a}}+h_{\mathrm{a}}\right) N \\
0 & =r\left(R_{\max }-R\right)-I_{\max } \frac{R}{H+R}\left(\int_{s_{0}}^{s_{\mathrm{m}}} s n(s) d s+s_{\mathrm{m}} q N\right) .
\end{aligned}
$$


Note that by assumption $w_{\mathrm{j}}(R)>0$. Integrating both sides of the first equation of (13) and solving for $n(s)$ gives

$$
n(s)=n\left(s_{0}\right)\left(\frac{s}{s_{0}}\right)^{-\left(1+\frac{d_{\mathbf{j}}+h_{\mathrm{j}}}{w_{\mathrm{j}}(R)}\right)} .
$$

Substituting (14) into the third equation of (13) yields

$$
\left(d_{\mathrm{a}}+h_{\mathrm{a}}\right) N=n\left(s_{0}\right) s_{\mathrm{m}} w_{\mathrm{j}}(R)\left(\frac{s_{\mathrm{m}}}{s_{0}}\right)^{-\left(1+\frac{d_{\mathrm{j}}+h_{\mathrm{j}}}{w_{\mathrm{j}}(R)}\right)} .
$$

From the second equation of (13) and (15), we have

$$
f(R) \stackrel{\text { def }}{=} \frac{w_{\mathrm{a}}(R)}{\left(d_{\mathrm{a}}+h_{\mathrm{a}}\right)}\left(\frac{s_{\mathrm{m}}}{s_{0}}\right)^{1-\frac{d_{\mathrm{j}}+h_{\mathrm{j}}}{w_{\mathrm{j}}(R)}}=1 .
$$

Since $f(R)$ is a monotonically increasing with respect to $R$, Eq. (16) has at most one positive root $\mathcal{R}, R_{\mathrm{jT}}<\mathcal{R}<R_{\max }$, independent of $n\left(s_{0}\right)$. Using (14), we see that the total biomass in the juvenile stage at equilibrium is given by

$$
\mathcal{J}=\int_{s_{0}}^{s_{\mathrm{m}}} s n(s) d s=\frac{n\left(s_{0}\right) s_{0}^{2}}{1-\frac{d_{\mathrm{j}}+h_{\mathrm{j}}}{w_{\mathrm{j}}(R)}}\left(\left(\frac{s_{\mathrm{m}}}{s_{0}}\right)^{1-\frac{d_{\mathrm{j}}+h_{\mathrm{j}}}{w_{\mathrm{j}}(R)}}-1\right) .
$$

Solving the second equation of system (13) for $N$ at equilibrium yields

$$
\mathcal{N}=\frac{n\left(s_{0}\right) s_{0}^{2} w_{\mathrm{j}}(R)}{\left(d_{\mathrm{a}}+h_{\mathrm{a}}\right) s_{\mathrm{m}}}\left(\frac{s_{\mathrm{m}}}{s_{0}}\right)^{1-\frac{d_{\mathrm{j}}+h_{\mathrm{j}}}{w_{\mathrm{j}}(R)}}
$$

and the total biomass in the adult stage at equilibrium is given by $\mathcal{A}=s_{\mathrm{m}} \mathcal{N}$. Substituting (17) and (18) into the fourth equation of (13) gives

$$
\begin{aligned}
r\left(R_{\max }-R\right) & -I_{\max } \frac{R}{H+R}\left[\frac{n\left(s_{0}\right) s_{0}^{2}}{1-\frac{d_{\mathrm{j}}+h_{\mathrm{j}}}{w_{\mathrm{j}}(R)}}\left(\left(\frac{s_{\mathrm{m}}}{s_{0}}\right)^{1-\frac{d_{\mathrm{j}}+h_{\mathrm{j}}}{w_{\mathrm{j}}(R)}}-1\right)\right. \\
& \left.+\frac{q n\left(s_{0}\right) s_{0}^{2} w_{\mathrm{j}}(R)}{d_{\mathrm{a}}+h_{\mathrm{a}}}\left(\frac{s_{\mathrm{m}}}{s_{0}}\right)^{1-\frac{d_{\mathrm{j}}+h_{\mathrm{j}}}{w_{\mathrm{j}}(R)}}\right]=0 .
\end{aligned}
$$

Equation (19) has a unique positive root $n\left(s_{0}\right)$ for $R$ given, $R_{\mathrm{jT}}<R<R_{\max }$. Once $\mathcal{R}$ and $n\left(s_{0}\right)$ have been solved from (16) and (19), the positive steady juvenile and adult biomass density $\mathcal{J}$ and $\mathcal{A}$ are obtained. Thus, system (1) has at most one positive equilibrium. This completes the proof of the theorem.

After deriving the stage-structured biomass model, System (9), De Roos et al. (2008) concluded that it has the same equilibria as System (1). For the sake of completeness, we provide a rigorous proof of this fact: 
Theorem 3.3. System (9) has exactly the same equilibria as System (1).

Proof. The extinction equilibrium $\left(0,0, R_{\max }\right)$ clearly exists for both systems.

Let $(\mathcal{J}, \mathcal{A}, \mathcal{R})$ be the unique interior equilibrium of System (1) guaranteed by Theorem 3.2. It follows by construction, exactly as in De Roos et al., 2008 [Eqs. (12)-(20)] that then $(\mathcal{J}, \mathcal{A}, \mathcal{R})$ is also an equilibrium of System (9).

Consider the following equilibrium equations of System (9)

$$
\begin{aligned}
w_{\mathrm{a}}(R) A+\left(w_{\mathrm{j}}(R)-v\left(w_{\mathrm{j}}(R)\right)-d_{\mathrm{j}}-h_{\mathrm{j}}\right) J & =0, \\
v\left(w_{\mathrm{j}}(R)\right) J-\left(d_{\mathrm{a}}+h_{\mathrm{a}}\right) A & =0, \\
r\left(R_{\max }-R\right)-I_{\max } \frac{R}{H+R}(J+q A) & =0 .
\end{aligned}
$$

Substituting the second equation of (20) into the first equation of (20) yields

$$
g(R) \stackrel{\text { def }}{=} \frac{w_{\mathrm{a}}(R) v\left(w_{\mathrm{j}}(R)\right)}{\left(d_{\mathrm{a}}+h_{\mathrm{a}}\right)\left[v\left(w_{\mathrm{j}}(R)\right)-w_{\mathrm{j}}(R)+d_{\mathrm{j}}+h_{\mathrm{j}}\right]}=1 .
$$

By plugging in $v\left(w_{\mathrm{j}}(R)\right)$ in $g(R)$ we see that $g(R)=f(R)$, where $f(R)$ is the function defined in (16). Hence, the existence of interior equilibria $R^{*}$ for the resource is equivalent for both systems. Once $R^{*}=\mathcal{R}$ has been solved from (21), the equilibrium juvenile and adult biomass density for System (9), $J^{*}$ and $A^{*}$, are uniquely obtained from (20) and yield

$$
J^{*}=\frac{r\left(R_{\max }-R^{*}\right)\left(H+R^{*}\right)\left(d_{\mathrm{a}}+h_{\mathrm{a}}\right)}{I_{\max } R^{*}\left(d_{\mathrm{a}}+h_{\mathrm{a}}+q v\left(w_{\mathrm{j}}\left(R^{*}\right)\right)\right)}, \quad A^{*}=\frac{r\left(R_{\max }-R^{*}\right)\left(H+R^{*}\right) v\left(w_{\mathrm{j}}\left(R^{*}\right)\right)}{I_{\max } R^{*}\left(d_{\mathrm{a}}+h_{\mathrm{a}}+q v\left(w_{\mathrm{j}}\left(R^{*}\right)\right)\right)} .
$$

Thus, there exists a unique positive equilibrium for System (9), and hence we must have $\left(J^{*}, A^{*}, R^{*}\right)=$ $(\mathcal{J}, \mathcal{A}, \mathcal{R})$. The theorem follows.

From the proofs of Theorems 3.2 and 3.3, we can obtain information about when the positive equilibrium exists. To do so, let

$$
\Theta\left(h_{\mathrm{j}}, h_{\mathrm{a}}\right) \stackrel{\text { def }}{=} \frac{w_{\mathrm{a}}\left(R_{\max }\right)}{d_{\mathrm{a}}+h_{\mathrm{a}}} \frac{v\left(w_{\mathrm{j}}\left(R_{\max }\right)\right)}{v\left(w_{\mathrm{j}}\left(R_{\max }\right)\right)-w_{\mathrm{j}}\left(R_{\max }\right)+d_{\mathrm{j}}+h_{\mathrm{j}}} .
$$

From (16) and (21), we prove the following corollary. Here and in the rest of the paper, $\left(J^{*}, A^{*}, R^{*}\right)$ denotes the unique positive equilibrium.

Corollary 3.1. System (1) and System (9) always has the extinction equilibrium $\left(0,0, R_{\max }\right)$. The unique positive equilibrium $\left(J^{*}, A^{*}, R^{*}\right)$ exists in both systems if and only if $\Theta\left(h_{j}, h_{a}\right)>1$ where $\Theta\left(h_{j}, h_{a}\right)$ is as in $(22)$.

\subsection{Local stability of equilibria}

In this section, we investigate the local stability of equilibria to the stage-structured population model, System (9). From local linearization, we obtain the following: 
Theorem 3.4. The extinction equilibrium $\left(0,0, R_{\max }\right)$ is locally stable if and only if $\Theta\left(h_{j}, h_{a}\right)<1$. The positive equilibrium $\left(J^{*}, A^{*}, R^{*}\right)$ is stable if $\Psi\left(h_{j}, h_{a}\right)>0$ with $\Psi\left(h_{j}, h_{a}\right)$ given by $(24)$.

For the parameters in Table 1 , we see numerically that $\Theta\left(h_{\mathrm{j}}, h_{\mathrm{a}}\right)>1$ implies $\Psi\left(h_{\mathrm{j}}, h_{\mathrm{a}}\right)>0$. Hence, for these parameters, the positive equilibrium is always locally stable.

Proof of Theorem 3.4. The stability of the extinction equilibrium $\left(0,0, R_{\max }\right)$ is governed by the following characteristic equation, that results from straight forward linearization:

$$
\left|\begin{array}{ccc}
w_{\mathrm{j}}\left(R_{\max }\right)-v\left(w_{\mathrm{j}}\left(R_{\max }\right)\right)-d_{\mathrm{j}}-h_{\mathrm{j}}-\lambda & w_{\mathrm{a}}\left(R_{\max }\right) & 0 \\
v\left(w_{\mathrm{j}}\left(R_{\max }\right)\right. & -d_{\mathrm{a}}-h_{\mathrm{a}}-\lambda & 0 \\
-I_{\max } \frac{R_{\max }}{H+R_{\max }} & -q I_{\max } \frac{R_{\max }}{H+R_{\max }} & -r-\lambda
\end{array}\right|=0,
$$

i.e.

$$
\lambda^{3}+a \lambda^{2}+b \lambda+c=0,
$$

where

$$
\begin{aligned}
a= & r+d_{\mathrm{j}}+h_{\mathrm{j}}+d_{\mathrm{a}}+h_{\mathrm{a}}+v\left(w_{\mathrm{j}}\left(R_{\max }\right)\right)-w_{\mathrm{j}}\left(R_{\max }\right)>0 \\
b= & \left(d_{\mathrm{a}}+h_{\mathrm{a}}\right)\left[v\left(w_{\mathrm{j}}\left(R_{\max }\right)\right)+d_{\mathrm{j}}+h_{\mathrm{j}}-w_{\mathrm{j}}\left(R_{\max }\right)\right]-w_{\mathrm{a}}\left(R_{\max }\right) v\left(w_{\mathrm{j}}\left(R_{\max }\right)\right), \\
& +r\left[d_{\mathrm{j}}+h_{\mathrm{j}}+d_{\mathrm{a}}+h_{\mathrm{a}}+v\left(w_{\mathrm{j}}\left(R_{\max }\right)\right)-w_{\mathrm{j}}\left(R_{\max }\right)\right], \\
c= & r\left(d_{\mathrm{a}}+h_{\mathrm{a}}\right)\left[d_{\mathrm{j}}+h_{\mathrm{j}}+v\left(w_{\mathrm{j}}\left(R_{\max }\right)\right)-w_{\mathrm{j}}\left(R_{\max }\right)\right]+r w_{\mathrm{a}}\left(R_{\max }\right) v\left(w_{\mathrm{j}}\left(R_{\max }\right)\right) .
\end{aligned}
$$

Applying the Routh-Hurwitz criterion, which states that all roots of (23) have negative real parts if and only if $a>0, c>0$ and $a b>c$, results in the conclusion $\left(0,0, R_{\max }\right)$ is locally stable if and only if $\Theta\left(h_{\mathrm{j}}, h_{\mathrm{a}}\right)<1$.

The stability of the positive equilibrium $\left(J^{*}, A^{*}, R^{*}\right)$ of System (9) is governed by

$$
\left|\begin{array}{ccc}
a_{11}-\lambda & a_{12} & a_{13} \\
a_{21} & a_{22}-\lambda & a_{23} \\
a_{31} & a_{32} & a_{33}-\lambda
\end{array}\right|=0,
$$

in which

$$
\begin{gathered}
a_{11}=-\frac{w_{\mathrm{a}}\left(R^{*}\right) A^{*}}{J^{*}}, a_{12}=w_{\mathrm{a}}\left(R^{*}\right), a_{13}=\frac{\partial w_{\mathrm{a}}\left(R^{*}\right)}{\partial R} A^{*}+\frac{\partial w_{\mathrm{j}}\left(R^{*}\right)}{\partial R} J^{*}-\frac{\partial v\left(w_{\mathrm{j}}\left(R^{*}\right)\right)}{\partial R} J^{*}, \\
a_{21}=v\left(w_{\mathrm{j}}\left(R^{*}\right)\right), a_{22}=-d_{\mathrm{a}}-h_{\mathrm{a}}, a_{23}=\frac{\partial v\left(w_{\mathrm{j}}\left(R^{*}\right)\right)}{\partial R} J^{*}, \\
a_{31}=-I_{\max } \frac{R^{*}}{H+R^{*}}, a_{32}=q a_{31}, a_{33}=-r-\frac{H I_{\max }\left(J^{*}+q A^{*}\right)}{\left(H+R^{*}\right)^{2}},
\end{gathered}
$$

i.e.

$$
\lambda^{3}+a \lambda^{2}+b \lambda+c=0,
$$


where

$$
\begin{aligned}
& a=-\left(a_{11}+a_{22}+a_{33}\right)>0, \\
& b=a_{22} a_{33}-a_{23} a_{32}+a_{11} a_{33}+a_{13} a_{31}, \\
& c=a_{11} a_{23} a_{32}+a_{13} a_{31} a_{22}-a_{12} a_{23} a_{31}-a_{13} a_{21} a_{32}>0 .
\end{aligned}
$$

Applying the Routh-Hurwitz criterion once more, we see that since $a, c>0,\left(J^{*}, A^{*}, R^{*}\right)$ is stable if and only if

$$
\Psi\left(h_{\mathrm{j}}, h_{\mathrm{a}}\right) \stackrel{\text { def }}{=} a b-c>0 .
$$

This completes the proof of Theorem 3.4.

By tracing $\left(J^{*}, A^{*}, R^{*}\right)$ with the bifurcation software AUTO, and by Theorem 3.4 above, we believe the following holds: If we allow $A, J, R \in \mathbf{R}$, then both equilibria exists near $\Theta=\Theta\left(h_{\mathrm{j}}, h_{\mathrm{a}}\right)=1$ and they meet at a transcritical bifurcation at $\left(0,0, R_{\max }\right)$ when $\Theta=1$. Hence, the stability of the two equilibria is exchanged when they collide. So $\left(J^{*}, A^{*}, R^{*}\right)$ is stable and $\left(0,0, R_{\max }\right)$ is unstable when $\Theta>1$, and vice versa when $\Theta<1$. See Sect. 3.5 for details.

\subsection{The stock recovery potential}

The quantity $\Theta=\Theta\left(h_{\mathrm{j}}, h_{\mathrm{a}}\right)$ defined by (22), which appears in Theorem 3.4, is the generational net biomass production (per unit body mass) in a virgin environment, i.e., when the resource biomass is close to its maximum value $R_{\max }$. In other words, $\Theta$ approximates the net reproduction (expressed in biomass) of a population small enough to have a negligible effect on the resource biomass, which means that $\Theta>1$ ensures that the biomass of an initially small population will increase in the absence of demographic stochasticity, while $\Theta<1$ ensures that the population will go extinct. Hence, $\Theta$ can be seen as a measure of the potential of a stock to recover from a small population size. Therefore, we refer to $\Theta$ as the recovery potential of a population. The recovery potential gives an indication of a populations productivity and stability, and a value close to unity reveals that the population is sensitive to disturbances. Typically, increased harvesting will lower a stocks recovery potential.

The measure of recovery potential which we use here is closely related to, and could likely be replaced by, the basic reproductive ratio $\left(R_{0}\right)$ in a virgin environment. The main difference is that our measure of recovery potential is based on growth in biomass, whereas $R_{0}$ is based on growth in the number of individuals.

\subsection{Numerical investigation of the basin of attraction}

We present numerical investigations indicating that the locally stable equilibria in System (9) are in fact globally stable. The numerical investigations are performed as follows: For fixed parameters 
and different initial conditions in the $J A R$-space, the trajectory is examined to see if it converges to the same equilibrium, independent of the initial conditions.

For the parameters $h_{\mathrm{j}}$ and $h_{\mathrm{a}}$, we consider the grid

$$
h_{\mathrm{j}} \in[0,0.2,0.4, \ldots, 4], \quad h_{\mathrm{a}} \in[0,5,10, \ldots, 140] .
$$

Concerning initial conditions, we let the trajectory start at points in the grid

$$
J, A \in[0,0.2,0.4, \ldots, 1]+\epsilon, \quad R \in[0,0.4,0.8, \ldots, 2],
$$

with $\epsilon=0.01$. We integrate System (9) from time $t=0$ to time $t=T$, and then we check if $\left|(J, A, R)-\left(J^{*}, A^{*}, R^{*}\right)\right| \leq 0.01$. It turns out that if we chose $T$ large enough, then as expected, all trajectories for the same parameter values end up at the same equilibrium. Hence, this indicates that the locally stable equilibria are global attractors. For all tested points in the $h_{\mathrm{j}} h_{\mathrm{a}}$-plane below the extinction boundary, given by $\Theta=1$, trajectories end up at the interior equilibrium $\left(J^{*}, A^{*}, R^{*}\right)$, and for points above the extinction boundary, trajectories end up at $\left(0,0, R_{\max }\right)$. Hence, below the extinction boundary, the interior equilibrium $\left(J^{*}, A^{*}, R^{*}\right)$ seems to be the global attractor, and above the curve the population seems to die out, i.e., $\left(0,0, R_{\max }\right)$ is the global attractor.

To investigate the possibility of the existence of more attractors such as periodic solutions, we have carried out additional AUTO investigations. In particular, we have traced the interior equilibrium in the parameters $h_{\mathrm{j}}, h_{\mathrm{a}}$, indicating that the equilibria do not bifurcate outside the curve $\Theta\left(h_{\mathrm{j}}, h_{\mathrm{a}}\right)=1$. This indicates that there are no more attractors in the system.

\section{Management of stage-structured fish stocks}

Classical management theory, which aims to maximize yield or profit, usually studies size-independent harvesting for which the harvesting effort can be represented by a single real number (see e.g. Clark, 2010). However, for structured populations under size-dependent harvesting there can be several different harvesting strategies which give the same, or similar, yield or profit, but differ significantly from an ecological perspective, e.g. in the risk of extinction.

In Sect. 3, we introduced the stock recovery potential $\Theta$ which represents the net generational biomass production (per unit body mass) in a virgin environment. This quantity allows a natural interpretation as the ability to recover from overfishing. In particular, a higher value of $\Theta$ makes the population less vulnerable to random extinction following disturbances. Hence, the value of $\Theta$ associated with a harvesting strategy gives an indication of how risky the harvesting strategy is from an extinction perspective.

To manage a fishery sustainably, we wish to ensure the harvested species' long-term survival in addition to achieving high yield of profit. We account for the extinction risk associated with a 
harvesting strategy by incorporating the recovery potential as a policy objective. Thus, our aim is to maximize the target (yield or profit) while simultaneously minimizing the ecological risk. This is a multi-objective optimization problem which lacks a unique solution as the target trades-off against the recovery potential. It is, however, natural to consider a strategy to be inefficient if there is another strategy that can achieve an equal or better value for both the target and the recovery potential. By removing all such inefficient strategies, we are left with a subset of strategies that can be identified with the so-called Pareto efficient strategies, also called the Pareto front.

\subsection{Methodological underpinnings}

We consider two harvesting targets: yield and profit at the interior equilibrium as determined by the following functions,

$$
P\left(h_{\mathrm{j}}, h_{\mathrm{a}}\right)=p_{\mathrm{j}} h_{\mathrm{j}} J^{*}\left(h_{\mathrm{j}}, h_{\mathrm{a}}, R^{*}\left(h_{\mathrm{j}}, h_{\mathrm{a}}\right)\right)+p_{\mathrm{a}} h_{\mathrm{a}} A^{*}\left(h_{\mathrm{j}}, h_{\mathrm{a}}, R^{*}\left(h_{\mathrm{j}}, h_{\mathrm{a}}\right)\right)-c_{\mathrm{j}} h_{\mathrm{j}}-c_{\mathrm{a}} h_{\mathrm{a}},
$$

and

$$
Y\left(h_{\mathrm{j}}, h_{\mathrm{a}}\right)=h_{\mathrm{j}} J^{*}\left(h_{\mathrm{j}}, h_{\mathrm{a}}, R^{*}\left(h_{\mathrm{j}}, h_{\mathrm{a}}\right)\right)+h_{\mathrm{a}} A^{*}\left(h_{\mathrm{j}}, h_{\mathrm{a}}, R^{*}\left(h_{\mathrm{j}}, h_{\mathrm{a}}\right)\right) .
$$

Here, $p_{\mathrm{j}}$ and $p_{\mathrm{a}}$ are the respective prices of juvenile and adult fish, while $c_{\mathrm{j}}$ and $c_{\mathrm{a}}$ are the respective costs of harvesting juvenile fish and adult fish. In particular, if $c_{\mathrm{j}}=c_{\mathrm{a}}=0$ and $p_{\mathrm{j}}=p_{\mathrm{a}}=1$, then $P\left(h_{\mathrm{j}}, h_{\mathrm{a}}\right)$ equals the yield objective function $Y\left(h_{\mathrm{j}}, h_{\mathrm{a}}\right)$.

For the given target (yield or profit), we aim to minimize the ecological risk by choosing an harvesting strategy that maximizes the recovery potential. For this purpose, we define an optimal harvesting strategy as follows.

Definition 4.1. The harvesting strategy $\left(\widehat{h_{j}}, \widehat{h_{a}}\right)$ is optimal for yield $Y\left(\widehat{h_{j}}, \widehat{h_{a}}\right)$ if for all $\left(h_{j}, h_{a}\right)$ such that $Y\left(h_{j}, h_{a}\right)=Y\left(\widehat{h_{j}}, \widehat{h_{a}}\right)$ we have $\Theta\left(h_{j}, h_{a}\right) \leq \Theta\left(\widehat{h_{j}}, \widehat{h_{a}}\right)$. Optimal harvesting for profit is defined as above but with $Y\left(h_{j}, h_{a}\right)$ replaced by $P\left(h_{j}, h_{a}\right)$.

The respective catch levels which result when the two targets, yield and profit, are maximized independent of the ecological cost are commonly referred to as maximum sustainable yield (MSY) and maximum economic yield (MEY), respectively. Christiansen (2009) argues that the former is the better target for society as a whole, while the latter is a natural target for a fishing fleet. We consider a continuum of harvesting strategies connecting MSY with MEY. Specifically, we consider harvesting strategies in the sets

$$
D=\left\{\left(h_{\mathrm{j}}, h_{\mathrm{a}}\right): \Theta\left(h_{\mathrm{j}}, h_{\mathrm{a}}\right)>1 \text { and } h_{\mathrm{j}}, h_{\mathrm{a}} \geq 0\right\},
$$

and

$$
\mathrm{L}_{\mathrm{EB}}=\left\{\left(h_{\mathrm{j}}, h_{\mathrm{a}}\right): \Theta\left(h_{\mathrm{j}}, h_{\mathrm{a}}\right)=1 \text { and } h_{\mathrm{j}}, h_{\mathrm{a}} \geq 0\right\} .
$$


Here, $\mathrm{L}_{\mathrm{EB}}$ is the extinction boundary. The region $D$ consists of the viable harvesting strategies, where each harvesting strategy describes the effort allocation on juveniles and adults.

Note that there exist harvesting strategies which maximize the target functions $Y\left(h_{\mathrm{j}}, h_{\mathrm{a}}\right)$, $P\left(h_{\mathrm{j}}, h_{\mathrm{a}}\right)$, and $\Theta\left(h_{\mathrm{j}}, h_{\mathrm{a}}\right)$ since these functions are continuous in the closed set $D \cup \mathrm{L}_{\mathrm{EB}}$. Hence, to find an optimal strategy to catch a given amount of yield $Y_{0}$, we solve the optimization problem

$$
\max _{\left(h_{\mathrm{j}}, h_{\mathrm{a}}\right) \in E} \Theta\left(h_{\mathrm{j}}, h_{\mathrm{a}}\right), \quad \text { where } \quad E=\left\{\left(h_{\mathrm{j}}, h_{\mathrm{a}}\right) \mid Y\left(h_{\mathrm{j}}, h_{\mathrm{a}}\right)=Y_{0}\right\} .
$$

Optimal harvesting with respect to yield and profit then represents two harvesting management methods, which we refer to as yield management and profit management, respectively.

\subsection{Pareto-optimal harvesting strategies}

Figure 1(a)-(c) shows Pareto plots for all pairwise combinations of the three harvesting targets yield, profit, and recovery potential. The Pareto efficient strategies are indicated with part of colored curves as described in the figure caption. The points $\mathrm{M}_{\mathrm{MSY}}$ and $\mathrm{C}_{\mathrm{MEY}}$ correspond to the harvesting targets maximum sustainable yield (MSY) and maximum economic yield (MEY).

For a fixed yield (profit), the recovery potential is maximized (thus minimizing the ecological risk) on the Pareto front for yield (profit) in Fig. 1(a) (Fig. 1(b)). Therefore, the optimal harvesting strategies for yield management is described by the Pareto front in Fig. 1(a) and shown as the red and green curves from the point $\Theta(0,0)$ to the point $\mathrm{M}_{\mathrm{MSY}}$ in Fig. 1(b) and from the point $(0,0)$ to point $\mathrm{M}_{\mathrm{MSY}}$ in Fig. 1(d). The optimal harvesting strategies for profit management correspond to the Pareto front in Fig. 1(b) and shown as the blue curve from the point $\Theta(0,0)$ to the point $C_{M E Y}$ in Fig. $1(b)$ and from the point $(0,0)$ to point $C_{M E Y}$ in Fig.1(d). For profit management only adults are harvested. However, for yield management juveniles may be harvested.

Let us first consider yield management, and analyze the differences between the two targets MSY and MEY. Note that the recovery potential decreases as the landed yield increases. Therefore, since MEY is always lower than MSY, targeting MSY is riskier than targeting MEY, and landing a yield very close to MSY greatly increase the risk of extinction (Fig. 1(a)), while landing a yield close to MEY will be a red strategy on the Pareto front which gives a negative profit (red strategies in Fig. 1(b)). However, by landing a yield above MEY, but slightly below MSY (green curve in Fig. 1(a)), it is possible to turn the loss into a profit without reducing the recovery potential to very low levels. In fact, the peak profit is close to maximum, while the recovery potential of the strategy that gives the peak profit is significantly higher than the recovery potential for MSY (green curve in $1(\mathrm{~b})$ ).

Next consider profit management. Similarly as before, the recovery potential decreases as profit increase along the Pareto front. Since MEY is always lower than MSY, this choice of management method is safer from an ecological perspective but always produces a lower yield than with yield 
(a)

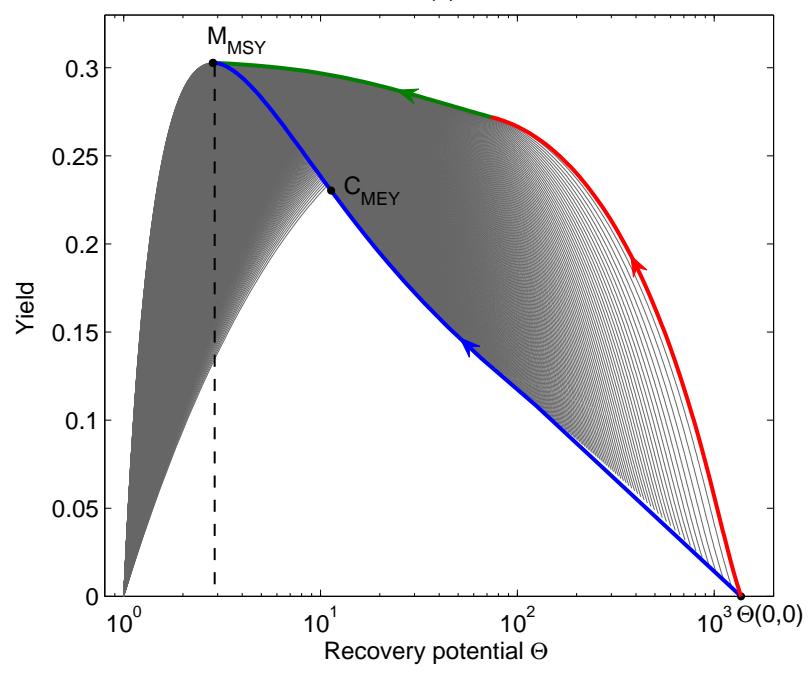

(c)

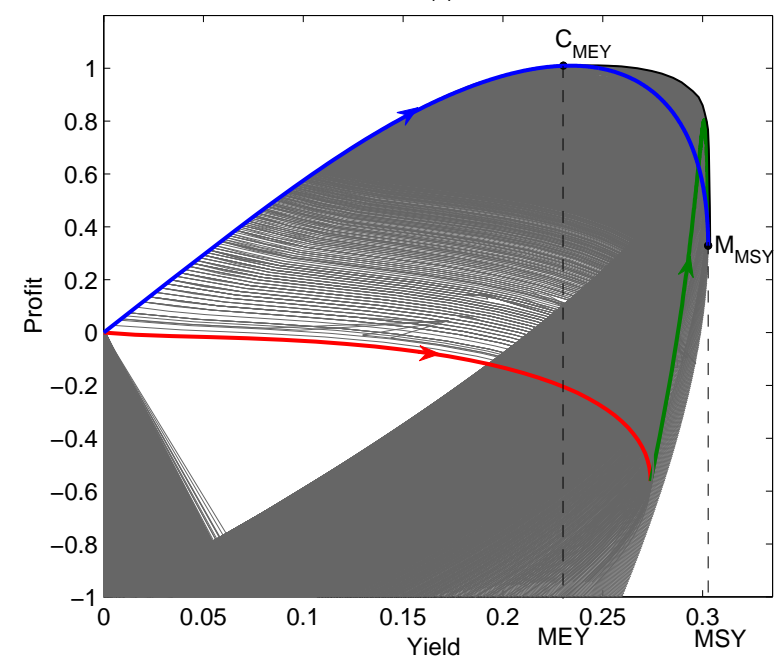

(b)

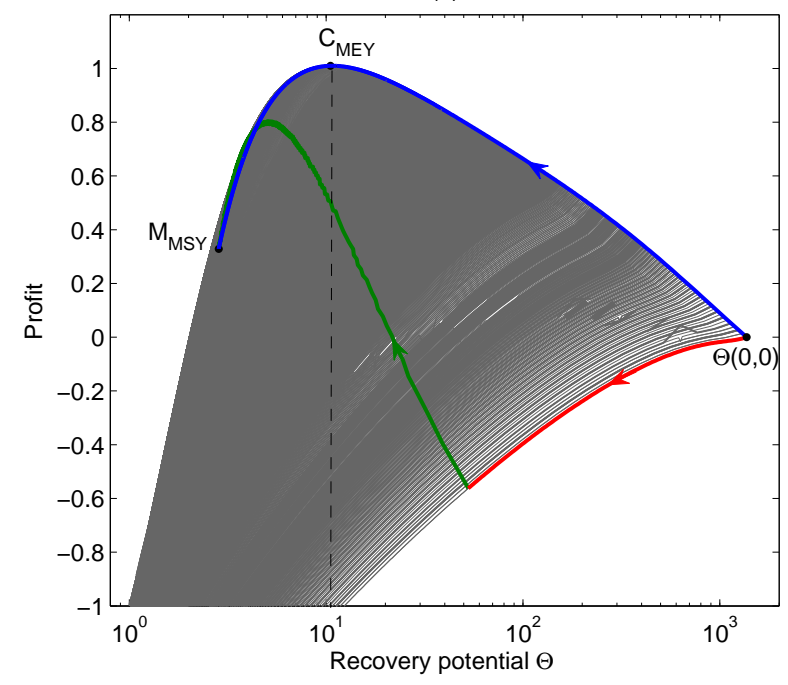

(d)

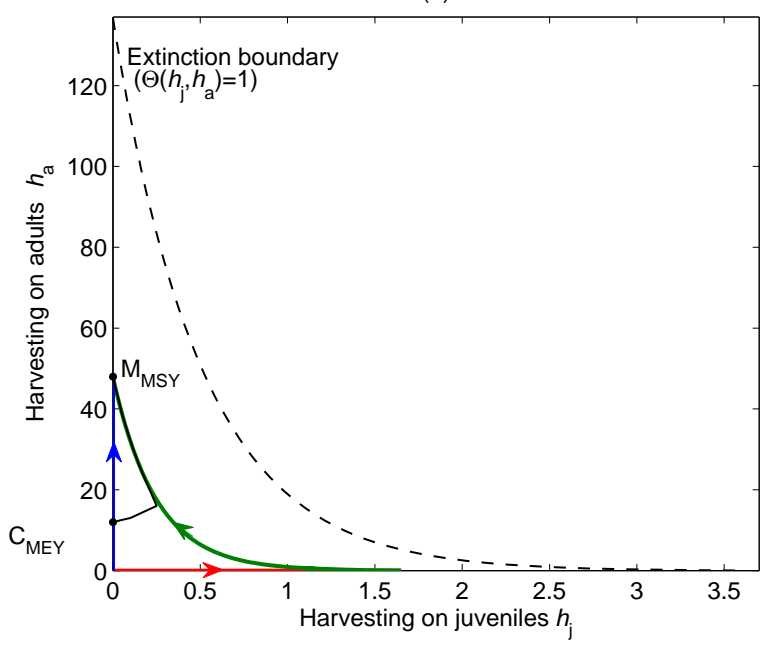

Figure 1: (a) Pareto plot for yield and recovery potential, in which optimal harvesting with respect to yield is given by the Pareto front, which consists of the red and green curves. (b) Pareto plot for profit and recovery potential, in which optimal harvesting with respect to profit is given by the Pareto front, which is the blue curve to the right of the dashed line. (c) Pareto plot for profit and yield, where the black curve from $\mathrm{C}_{\mathrm{MEY}}$ to $\mathrm{M}_{\mathrm{MSY}}$ is the Pareto front. (d) The Paretoefficient harvesting strategies for panels (a), (b), and (c). The green and blue curve corresponds to harvesting only juveniles and adults, respectively, while the green curve corresponds to mixed harvesting strategy encompassing both juveniles and adults. In panel (a)-(c), the point $\mathrm{C}_{\mathrm{MEY}}$ represents the mapping of the harvesting strategy that gives maximizes profit, and the point $\mathrm{M}_{\mathrm{MSY}}$ the mapping of the strategy that maximizes yield. The harvest rates of juveniles and adults vary, while all other parameters are as in Table 1. (Color figure online.) 
management for the same recovery potential. In fact, the strategy which maximizes profit for a given recovery potential simultaneously minimizes the yield. However, targeting MSY would result in a loss of more than half of the profit, while aiming for the maximum profit that can be achieved leads to the yield MEY which is significantly lower than MSY.

It is clear that yield and profit constitute conflicting targets, with or without taking recovery potential into consideration. This leads us to investigate the trade-off between yield and profit for different harvesting strategies, which we do by considering the Pareto efficient strategies for yield and profit in Fig. 1(c). Note that the recovery potential of the Pareto efficient strategies is always less than at MEY, but that the recovery potential is always higher than at MSY. Targeting MSY results in a significantly lower profit than targeting MEY. However, with only a small decrease in yield we can double the profit (the black curve from $\mathrm{C}_{\mathrm{MEY}}$ to $\mathrm{M}_{\mathrm{MSY}}$ in Fig. 1(c)). The green curve in Fig. 1(c) shows that this level of profit can be achieved with optimal strategies for yield management harvesting.

\subsection{The most and the least harmful harvesting strategy}

The distance to the extinction boundary (Fig. 1(d)) reflects the risk level of a harvesting strategy in the sense of sensitivity to changes in mortality rate, e.g., by increased harvesting or other external factors. This is because a small increase in mortality, e.g., due to illegal or unreported fishing, could make the strategy cross the extinction boundary. Applying the implicit function theorem on $\Theta\left(h_{\mathrm{j}}, h_{\mathrm{a}}\right)=1$, one can easily calculate the second derivative of $h_{\mathrm{a}}\left(h_{\mathrm{j}}\right)$ with respect with $h_{\mathrm{j}}$ :

$$
\frac{d^{2}}{d h_{\mathrm{j}}^{2}} h_{\mathrm{a}}\left(h_{\mathrm{j}}\right)=\frac{w_{\mathrm{a}}\left(R_{\max }\right) \ln ^{2}\left(\frac{s_{0}}{s_{\mathrm{m}}}\right)}{w_{\mathrm{j}}^{2}\left(R_{\max }\right)}\left(\frac{s_{\mathrm{m}}}{s_{0}}\right)^{1-\left(d_{\mathrm{j}}+h_{\mathrm{j}}\right) / w_{\mathrm{j}}(R)}>0,
$$

which shows that the graph of extinction boundary $\Theta\left(h_{\mathrm{j}}, h_{\mathrm{a}}\right)=1$ is convex upward (see $\mathrm{L}_{\mathrm{EB}}$ in Fig. 2). Therefore, the shortest distance to the extinction boundary $\mathrm{L}_{\mathrm{EB}}$ from any point in $D$ will be in the direction of a straight line that is not parallel to the axis, i.e., a harvest strategy that is a combination of harvesting both adults and juveniles. The slope of the line from the origin to curve $\mathrm{L}_{\mathrm{EB}}$ is

$$
k=\frac{w_{\mathrm{a}}\left(R_{\max }\right)\left(\frac{s_{\mathrm{m}}}{s_{0}}\right)^{1-\frac{d_{\mathrm{j}}+x^{*}}{w_{\mathrm{j}}\left(R_{\max }\right)}}}{x^{*}}
$$

where $x^{*}$ is a unique positive solution of the following equation with respect to variable $x$ :

$$
w_{\mathrm{a}}\left(R_{\max }\right)\left(\frac{s_{\mathrm{m}}}{s_{0}}\right)^{1-\frac{d_{\mathrm{j}}+x}{w_{\mathrm{j}}\left(R_{\max }\right)}} w_{\mathrm{a}}\left(R_{\max }\right)\left(\frac{s_{\mathrm{m}}}{s_{0}}\right)^{1-\frac{d_{\mathrm{j}}+x}{w_{\mathrm{j}}\left(R_{\max }\right)}} \ln \frac{s_{\mathrm{m}}}{s_{0}}-x w_{\mathrm{j}}\left(R_{\max }\right)=0 .
$$

For a non-harvested population, the shortest path from the origin to the extinction boundary curve $\mathrm{L}_{\mathrm{EB}}$ is in the direction of the line $h_{a}=k h_{\mathrm{j}}$, i.e., when the harvest effort is distributed between juveniles and adults as $(1, k) /(k+1)$ (see Fig. 2). This means that assuming a fixed harvest effort 
distribution, $(1, k) /(k+1)$ is the distribution that requires the least increase in total effort to cause the population to go extinct. In contrast, harvesting only adults requires the highest increase in total effort to cause extinction. Therefore, assuming a fixed harvest effort distribution, from an extinction perspective harvesting adults only is always the safest strategy, and harvesting juveniles is never the most harmful harvesting strategy. In general, the least harmful harvesting strategy will always be a single-stage harvesting strategy, i.e., harvesting either juveniles only or harvesting adults only, but which strategy it is may depend on the model parameters. However, we do not have an example of parameters for which harvesting adults are more harmful than harvesting juveniles.

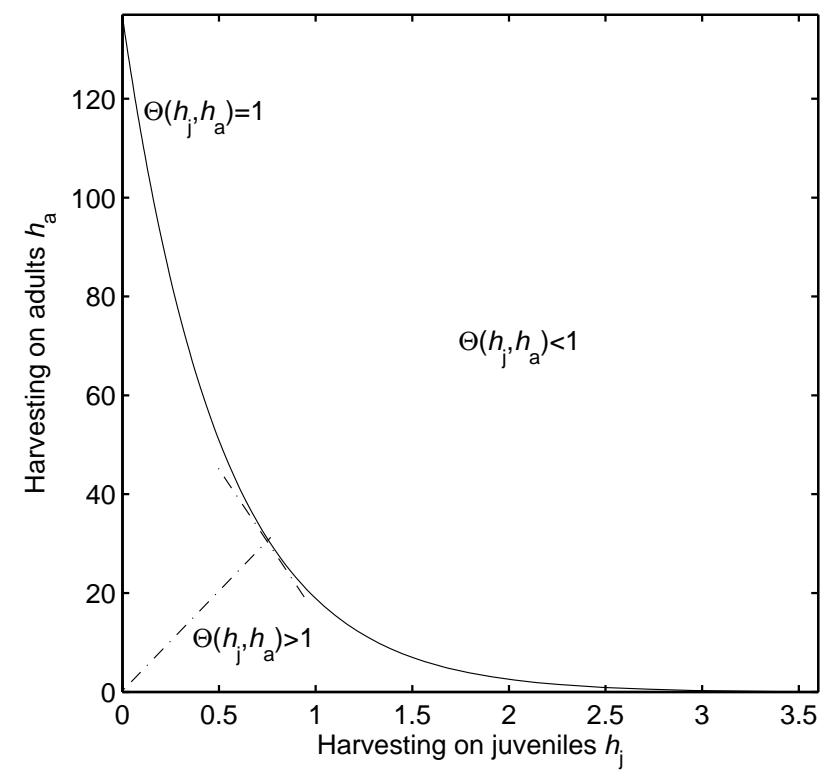

Figure 2: Assuming a fixed harvest effort distribution, the most harmful harvesting strategy from an extinction perspective is always a multi-stage harvesting strategy, i.e., simultaneously harvesting juveniles and adults, and the least harmful harvesting strategy is always a single-stage harvesting strategy. The reason is that the extinction boundary $\mathrm{L}_{\mathrm{EB}}$ given implicitly by $\Theta\left(h_{\mathrm{j}}, h_{\mathrm{a}}\right)=1$ is convex upward. The extinction region is given by $\Theta\left(h_{\mathrm{j}}, h_{\mathrm{a}}\right)<1$ and the sustainable harvesting region by $\Theta\left(h_{\mathrm{j}}, h_{\mathrm{a}}\right)>1$. From any point in the sustainable harvesting region, there exists a unique shortest path to the extinction boundary curve $\mathrm{L}_{\mathrm{EB}}$, which describes the harvest effort distribution that requires the least increase in total effort to cause the population to go extinct.

\subsection{Consequences of harvesting for population size-structure and biomass}

Field and experimental data indicate that size-dependent selective harvesting of large fish can result in a change in the size-distribution of the exploited stock toward smaller fish (Hamilton et 
al., 2007; Walsh et al., 2006). We investigate changes in population size-structure in response to stage-dependent harvesting of juveniles and adults.

Let $J^{f}$ and $A^{f}$ denote the fished juvenile biomass and adult biomass, respectively. The population size-structure is completely determined by the distribution of biomass between juveniles and adults and, therefore, we use the percentage of juveniles biomass $J^{f} /\left(J^{f}+A^{f}\right)$ to measure the impact of fishing on size-structure.

First, harvesting will induce a shift in size-composition towards juveniles relative the nonharvested population, that is, any type of fishing always results in a higher juvenile ratio in the fished population than in the non-fished population, as shown in Fig. 3(c). Harvesting adults affects the size-distribution faster and to a larger extent than what harvesting juveniles does (Fig. $3(\mathrm{a})-(\mathrm{b})$ ), and a high percentage of juvenile biomass indicates a low recovery potential, and thus a higher risk of extinction (Fig. 3(d)).

Second, the percentage of juvenile biomass in the population will initially increase when only juveniles are harvested, but will start to decrease as harvesting increases further, while the percentage of juveniles biomass can either increase or decrease if adults are harvested at a constant rate (Fig. 3(a)-(b)). Third, increasing the harvest rate of adult fish always induces an increase of the percentage of juveniles in the population (Fig. 3(b)), and Fig. 3 (a) and (b) show that harvesting juveniles and adults equally always leads to an increase in the percentage of juveniles in the population.

Third, for reproduction regulation $(q<1)$, De Roos et al. (2007) have shown that increased mortality may induce an increase in juvenile biomass, despite the fact that the total consumer biomass decreases. Juveniles use energy for growth, while adult individuals do not grow and instead use all the ingested energy for reproduction. Since juveniles have a higher ingestion ability, juveniles can compensate for the decrease in juvenile-stage biomass production from reproduction, as adult biomass decrease from harvesting, by growth in body size. This will lead to an increase in juvenile biomass although harvesting decreases the adult biomass (Fig. 1 in De Roos et al., 2007). In contrast, adult biomass always decreases with increasing harvest rates (Fig. 1 in De Roos et al., 2007). Hence, this shows that fishing leads to a higher percentage of juveniles irrespective of the type of fishing than without fishing, even if only juveniles are fished.

\subsection{Summary of main results}

We conclude by briefly summarizing our main results. (1) When the desired target is profit, only adults should be harvested, but when yield is the desired target, a small fraction of juveniles should be harvested as described by the green curve in Fig. 1(d). (2) In both cases, it is possible to increase the recovery potential considerably at a relatively small cost. This is particularly true when yield is the desired target. In this case, a reduction in the realized yield of only some $10 \%$ can lead to a tenfold increase in the recovery potential. (3) There exists a harvesting strategy 

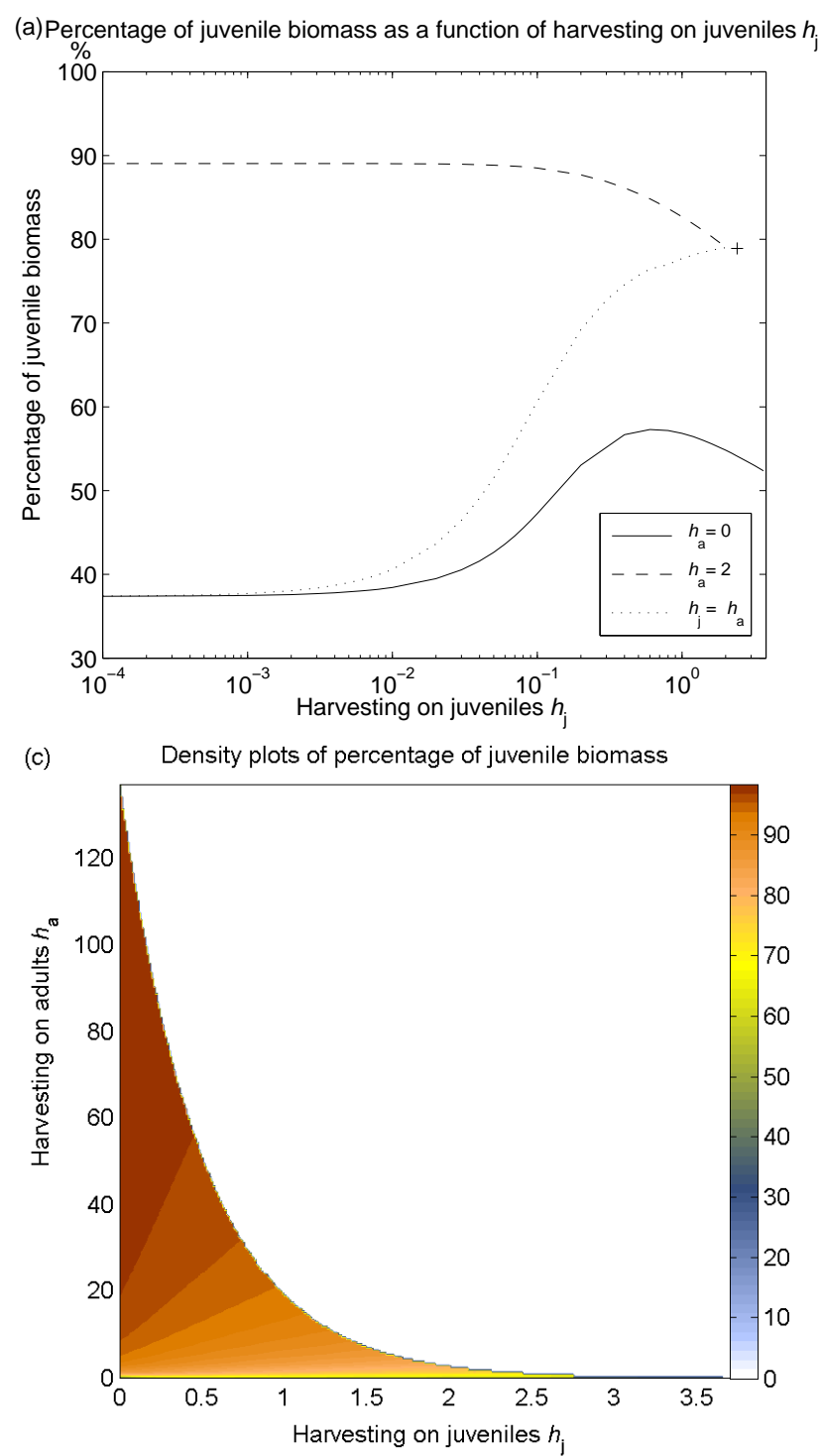

(b) Percentage of juvenile biomass as a function of harvesting on adults $h_{\mathrm{a}}$

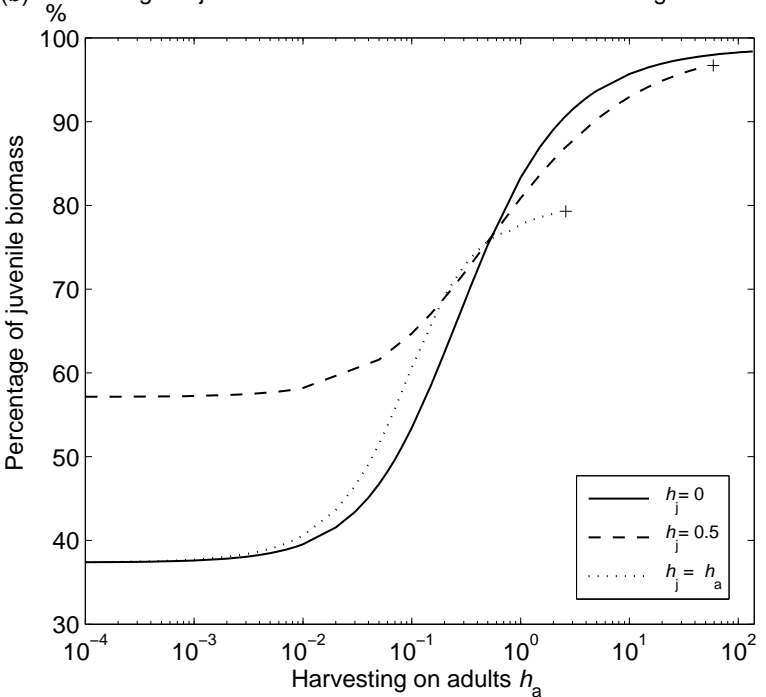

(d) Impact of Pareto optimal harvesting on percentage of juvenile biomass

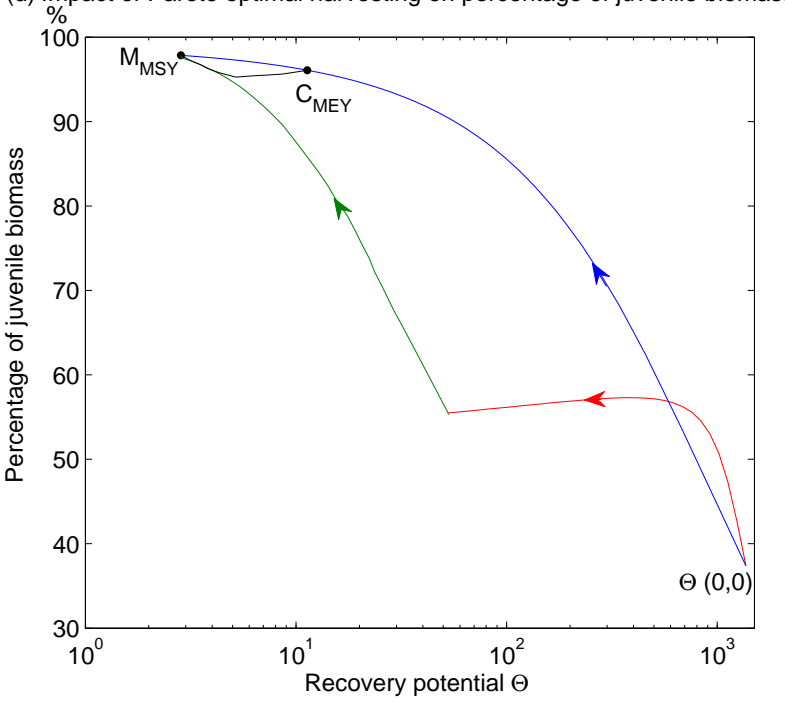

Figure 3: The effect of harvesting on population size structure: (a) Increasing harvesting on juveniles can either increase or decrease the proportion of juveniles in the population. (b) Increased harvesting on adults will always increase the proportion of juveniles in the population. (c) Harvesting will always increase the proportion of juveniles in the population relative the non-harvested population. (d) A high proportion of juveniles is an indication of low recovery potential. The harvest rates of juveniles and adults vary, while all other parameters are as in Table 1. (Color figure online.) 
which in both yield and profit is close to that associated with MSY and MEY. This strategy will be preferable over MSY or MEY in most instances. (4) The least harmful harvesting strategy is single-stage harvesting of either juveniles or adults (depending on stock dynamics) while the most harmful strategy consists of harvesting both juveniles and adults in some proportion. (5) A high precentage of juvenile biomass may be indicative of low recovery potential.

\section{Discussion}

We have analyzed dynamics and optimal management strategies in a stage-structured consumerresource fish stock model. First, we proved existence of equilibria and considered local stability. Numerical investigations of their basin of attraction indicate that the locally stable equilibria are global attractors. The question of proving global stability and absence of limit cycles in Systems (1) and (9) remains open. In order to explore optimal multi-objective harvesting strategies for the stage-structured population model, System (9), we introduced a measure which is relevant for sustainable harvesting - the recovery potential of a fish stock. The recovery potential reflects the ability of a population to grow from a small population size, i.e., the ability to recover from overfishing or any other major disturbance. Because the recovery potential acts as a measure of extinction risk, it allowed us to compare harvesting strategies from an ecological perspective.

To identify promising stage-dependent harvesting strategies, we numerically determined Pareto efficient strategies for the three possible pairs of yield, profit, and recovery potential. This led us to two important conclusions: First, a small decrease in the landed yield or profit relative to the maximum sustainable yield (MSY) or the profit associated with the maximum economic yield (MEY) allows for a disproportional increase in the fish stock's recovery potential. Second, there exists a harvesting strategy, which gives a yield close to MSY and a profit close to what is obtained at MEY. We believe that this harvesting strategy is preferable in most cases over targeting MSY or MEY alone. In examining the Pareto-efficient harvesting strategies, Fig. 2(d), we found that strategies optimal for a small yield targeted only juveniles, while strategies optimal for yields close to MSY included a proportion of adults. In contrast, strategies optimal for any level of profit exclusively targeted adults. We caution that these results are obtained for the specific parameter values given in Table 1. Changes in these parameters, and in particular the price of adult fish relative to juvenile fish, may have large impacts on our results. Moreover, our results do not account for induced evolutionary changes which can be undesirable (Olsen et al., 2004; Walsh et al., 2006).

Harvesting affects the size structure of fish stocks. For the specific parametrization used in our study, harvesting always resulted in an increase in the proportion of juveniles in the population, even when only juveniles were harvested. The fraction of juvenile biomass in the population appears to be negatively correlated with stock recovery potential. Consequently, a high percentage 
of juvenile biomass in the population may indicate an elevated risk of extinction. While this is in line with intuitive expectations, our findings highlight that size structure may be important for assessing the risk of extinction. Moreover, we would not be surprised if changes in stock size structure could serve as an early warning signal of an impending stock collapse.

In a separate analysis aimed at a broader audience, we intend to further develop the ideas presented here. Among other extensions, we will consider impact on stock size structure as an additional objective for Pareto efficiency and explore the extent to which the results depend on the mode of population regulation. Further work on sustainable harvesting of size- and stage-structure populations is highly desirable and could well result in effective tools for informing fisheries policy.

\section{Acknowledgments}

$\AA ̊ \mathrm{~B}$ and XM gratefully acknowledge financial support from the Wenner-Gren Foundations. We thank Lennart Persson for suggesting that we work with the simplified stage-structured model and two anonymous reviewers for their valuable comments that helped to improve the manuscript.

\section{References}

[1] Claessen, D., De Roos A.M., 2003. Bistability in a size-structured population model of cannibalistic fish-a continuation study. Theoretical Population Biology 64, 49-65.

[2] Clark, C.W., 2010. Mathematical Bioeconomics: The Mathematics of Conservation. 3th edition. New York:John Wiley and Sons, Wiley.

[3] Cushing, J.M., 1989. A competition model for size-structured species. SIAM Journal on Applied Mathematics 49, 838-858.

[4] De Roos, A.M., 1997. A gentle introduction to physiologically structured population models. In: Tuljapurkar, S., Caswell, H. (Eds.), Structured Population Models in Marine, Terrestrial and Freshwater Systems. Chapman-Hall, New York, pp. 119-204.

[5] De Roos, A.M., Schellekens, T., Van Kooten, T., De Wolfshaar, K.V,, Claessen, D., Persson, L., 2007. Food-Dependent Growth Leads to Overcompensation in Stage-Specific Biomass When Mortality Increases:The Influence of Maturation versus Reproduction Regulation. The American Naturalist 170(3), E59-E76.

[6] De Roos, A.M., Schellekens, T., Van Kooten, T., De Wolfshaar, K.V., Claessen, D., Persson, L., 2008. Simplifying a physiologically structured population model to a stage-structured biomass model. Theoretical Population Biology 73, 47-62.

[7] Diekmann, O., Metz, H., 1989. Exploring linear chain trickery for physiologically structured populations. In: Bij het afscheid van Prof. Dr. H.A. Lauwerier, TW in Beeld (Amsterdam: CWI), pp. 73-84.

[8] Farkas, J.Z., Hagen, T., 2007. Stability and regularity results for a size-structured population model. Journal of Mathematical Analysis and Applications 328, 119-136.

[9] Garcia, S.M., Newton C., 1995. Current situation, trends and prospects in world capture fisheries. Fisheries technical paper, Food and Agriculture Organization, Rome. 
[10] Hamilton, S.L., et al., 2007. Size-selective harvesting alters life histories of a temperate sex-changing fish. Ecological Applications 17(8), 2268-2280.

[11] Hutchings J.A., Myers R.A., 1994. What Can Be Learned from the Collapse of a Renewable Resource? Atlantic Cod, Gadus morhua, of Newfoundland and Labrador. Canadian Journal of Fisheries and Aquatic Sciences 51(9), 2126-2146.

[12] Metz, J.A.J., Diekmann, O., 1986. The Dynamics of Physiologically Structured Populations. Lecture Notes in Biomathematics, vol. 68, Springer, Heidelberg.

[13] Olsen, E.M., Heino, M., Lilly, G.R., Morgan, M.J., Brattey, J., Ernande, B. and Dieckmann, U., 2004. Maturation trends indicative of rapid evolution preceded the collapse of northern cod. Nature 428, 932-935.

[14] Persson, L., Leonardsson, K., De Roos, A.M., Gyllenberg, M., Christensen, B., 1998. Ontogenetic scaling of foraging rates and the dynamics of a size-structured consumer-resource model. Theoretical Population Biology 54, 270-293.

[15] Rijnsdorp, A.D., van Leeuwen, P.I., Daan, N., and Heesen, H.J.L., 1996. Changes in abundance of demersal fish species in the North Sea between 1906-1909 and 1990-1995, ICES Journal of Marine Science 53, 1054-1062.

[16] Walsh, M.R., Munch, S.B., Chiba, S., Conover, D.O., 2006. Maladaptive changes in multiple traits caused by fishing: impediments to population recovery. Ecology Letters 9, 142-148. 